\title{
Automatic Segmentation of Digital Orthopantomograms for Forensic Human Identification
}

\author{
Dariusz Frejlichowski and Robert Wanat \\ West Pomeranian University of Technology, Szczecin, \\ Faculty of Computer Science and Information Technology, \\ Zolnierska 52, 71-210, Szczecin, Poland \\ \{dfrejlichowski,rwanat\}@wi.zut.edu.pl
}

\begin{abstract}
Dental radiographic images are one of the most popular biometrics used in the process of forensic human identification. This led to the creation of the Automatic Dental Identification System with the goal of decreasing the time it takes to perform a single search in a large database of dental records. A fully automated system identifying people based on dental X-ray images requires a prior segmentation of the radiogram into sections containing a single tooth. In this paper, a novel method for such segmentation is presented, developed for the dental radiographic images depicting the full dentition - pantomograms. The described method utilizes the locations of areas between necks of teeth in order to determine the separating lines and does not depend on the articulation of gaps between adjacent teeth, thus improving the results achieved in the situation of severe occlusions.
\end{abstract}

Keywords: image segmentation, dental pantomography, dental human identification, ADIS, forensic identification.

\section{Introduction}

Human forensic identification is the process of determining the identity of a person using the evidence available in legal investigations. Teeth and bite are particularly popular for this application ([1]), as they are both robust to decomposition and highly discriminant (2]). In the wake of ever growing use of digital radiology, the Federal Bureau of Investigation (FBI) created the Dental Task Force (DTF) to improve the use of digital dental information in legal proceedings ([3]). One of its main tasks was the creation of an Automated Dental Identification System (ADIS) in order to preliminarily browse through a large database of dental X-ray images in the search for radiograms with the most similar characteristics to present in an input image. The features used for comparison in ADIS are morphologic, e.g. the shapes of individual teeth or their dental restorations. The model and functionality of ADIS were described in [4].

In the simplified model of ADIS ([5]), there are three preliminary steps before two radiograms are compared: image enhancement, image segmentation (separation of the image into regions containing at most one tooth) and feature 
extraction (finding the contour of the tooth). In this paper, a novel method for segmentation of panoramic dental X-rays is proposed and presented. Panoramic $\mathrm{X}$-rays, or pantomograms, are a type of extraoral radiograms (i.e. radiograms, where the film is located outside of the patient's mouth) showing the full dentition on a single image. A sample pantomogram (all pantomograms in this paper are presented courtesy of Pomeranian University of Medicine in Szczecin) can be seen in Fig. 1. To our best knowledge, few segmentation algorithms were developed solely for the use with pantomograms (see Section 2). Panoramic images contain the largest amount of information about dentition, but due to the rendering 3-dimensional, semi-circular geometry of the jaw onto a 2-dimensional image, the teeth presented on pantomograms have a tendency of occluding with each other.

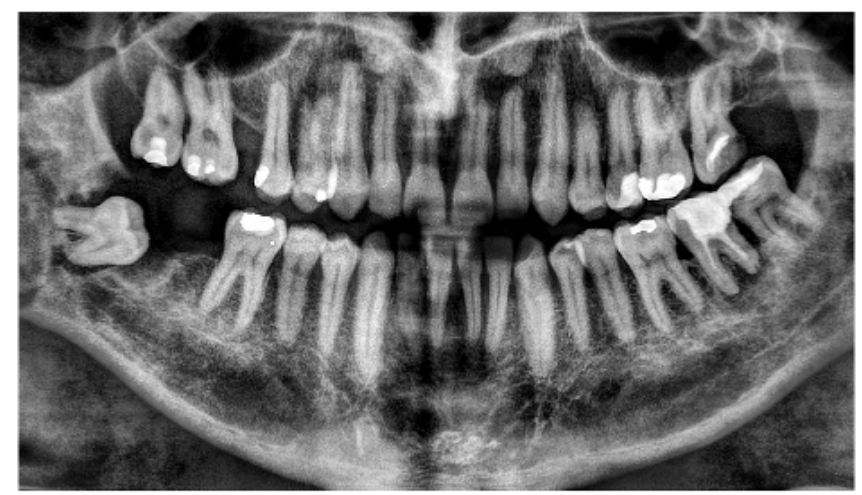

Fig. 1. A sample pantomogram after image enhancement and cropping

\section{Existing Segmentation Methods}

As has been mentioned in the previous section, there are several existing methods for dental radiogram segmentation. Most of these methods have been created with intraoral images in mind.

The first method was presented by Jain and Chen in [6]. It is focused on the application of the integral projections of pixels for the detection of gaps between teeth. The algorithm is separated into two parts: the first is the detection of the gap between lower and upper jaw, and the second is the detection of gaps separating individual teeth. The former step needs user input, so it can be considered semi-automatic. After the initial point of the gap has been selected by the user, moving in both directions, the algorithm chooses short horizontal lines with the highest probability of belonging to the gap. The probability is calculated using the equation $([6])$ :

$$
p_{v_{i}}\left(D_{i}, y_{i}\right)=p_{v_{i}}\left(D_{i}\right) p_{v_{i}}\left(y_{i}\right)
$$


where $p_{v_{i}}\left(D_{i}\right)$ is the normalized integral projection of a given horizontal line subtracted from 1 and $p_{v_{i}}\left(y_{i}\right)$ is a Gaussian with expected value equal to the position of the last chosen line (or the user selection in the first iteration). This probability function has its maximum for the horizontal line that is vertically close to the last selected line and that is composed of pixels with low values. After the maxima have been found for the whole image, a spline function is applied to form a smooth line that becomes the separating line between upper and lower jaw. Once the spline has been calculated, for every point on the curve a new integral projection is calculated in the direction perpendicular to its local curvature. These projections obtain low values in areas between teeth, thus the search for gaps can be reduced to searching for valleys in the plot of the integrals. The areas between these three lines (gap between upper/lower jaw, two successive gaps between teeth) and the horizontal borders of the image become the segments used later in the process of feature extraction. An improved version of this method was proposed in 7. In this case, the images are processed through wavelet kernels before calculating integral projections to further accentuate the gaps between the necks of teeth.

Another method, presented in [8], consists of the use of active contour models, also known as snakes. Initially described in [9], snakes are a model of parametrized splines driven towards edges and lines on the image by external forces, i.e. forces derived from the image on which they operate, as well as internal forces, i.e. user imposed control over the elasticity and rigidity of the contour. In [8], the external driving force along the contour of the snake $E_{e x t}$ is given as:

$$
E_{\text {ext }}(x, y)=G_{\sigma}(x, y) * I(x, y),
$$

where $G_{\sigma}$ is a 2-dimensional Gaussian and $I(x, y)$ is the original image. Thus defined external force amounts to the intensities Gaussian-filtered original image, in which case it takes the lowest values in the dark areas of the radiogram, such as the gap between upper and lower jaw or in spaces between teeth. When using properly selected initial approximations of these curves it achieves very good segmentation of the image.

\section{Description of the Proposed Method}

\subsection{Preliminary Steps}

For the proper work of the proposed method it is assumed that before an image is segmented using the method, it had been enhanced using the algorithm presented in 10]. That method is based on the decomposition of the image into a Laplacian pyramid, separating the radiogram into smaller images containing progressively lower frequencies of the signal present in the original image. Then, a range of simple filters is applied to selected layers of the pyramid, including sharpening filter and contrast enhancement methods, before the image is recomposed again. It is also necessary to locate the gap between the frontal teeth before the segmentation. In this paper, a nose position detection is used and then a vertical line on the same position is considered the center. 


\subsection{Separating the Upper and Lower Jaw}

The first step is the determination of the line separating the upper and lower jaw. The same method as presented in [6] and briefly described in the previous section is used. In order to automatize the process of segmentation, instead of requiring that the user inputs the initial separating point used by the algorithm, it is selected by choosing the horizontal integral projection around the center of the image with the lowest value, usually between $40 \%$ and $60 \%$ of the height of the image. Since the teeth on the image create an arc, instead of using the full horizontal line that would pass through teeth further from the incissors, only a small number of pixels (equal to $20 \%$ of the width of the image) closest to the previously selected frontal teeth gap is used to calculate the projections. The mentioned paramaters were established experimentally. Afterwards the algorithm proceeds as described in [6].

\subsection{Localization of the Areas between the Necks of Teeth}

The obtained curve is then used to estimate the position of the neck of every tooth that is the part of the tooth where roots end and the formation of crown and enamel begins. While the crowns of separate teeth tend to occlude with each other and roots are difficult to separate from the underlying bone, the area between the necks of two adjacent teeth is distinguishable enough to be easily found on a pantomogram. Since the necks of teeth are on the same height as dental pulp, which is darker than the surrounding teeth, the simplest method for finding a line going through dental necks is to translate the line separating upper and lower jaw vertically, sum the intensities of pixels the line passes through for every translation and select the ones for which there is a distinctive drop of values, indicating the line passes through darker areas of the pulp.

In order to guarantee that neither the original gap between jaws nor a gap between roots of teeth and the edge of the image are selected in lieu of the desired dental pulp line, the vertical translation scope should be chosen to conduct the search for a limited range, automatically discarding translations too close and too far from the line separating jaws. The result of this step are two values, one negative and one positive, that indicate the amount of pixels that the vertical position of every point belonging to the spline separating jaws should be moved for in order to receive the spline that passes through dental pulps of teeth in each jaw.

The next step is the selection of points on each spline representing a gap between the necks of two adjacent teeth. To refine the results of this stage of the algorithm, a new image is created by multiplying the value-inverted original image with local range filtered version of the original image. That image has high values for darker pixels that lie in areas with neighboring points of low and high intensity. For both upper and lower jaw, an array of values is saved containing the intensities of points belonging to the splines passing through their respective dental pulps. Sharp spikes on the plot of these values indicate 
dark spots surrounded by light regions, indicating a gap between necks of teeth. In order to remove false spikes, the values of the function are smoothed using the Gaussian filter. Then, starting from the previously selected line separating frontal teeth, small subsets of the values in the array are chosen for comparison. To determine the size of these subsets, average widths of teeth on every position were calculated based on twenty sample pantomograms. Both jaws are fairly symmetrical considering the size of teeth on a given position, thus only 8 values need to be calculated for each jaw, one for every tooth from first incissor to last molar.

To select the proper spike value indicating the gap between the necks of teeth for a currently searched tooth on the position $p_{c}$, a Bayesian probability $P\left(x_{i}, p_{c}\right)$ is calculated for each point on the curve $\left(x_{i}\right)$ according to the equation:

$$
P\left(x_{i}, p_{c}\right)=I\left(x_{i}\right) G\left(x_{i}, p_{c}\right) D\left(x_{i}, p_{c}\right),
$$

where $I$ is the intensity of the range filtered and inverted original image in the point $x_{i}$ and $G$ is a discrete Gaussian function with the expected value equal to the horizontal position of the last detected gap displaced left or right (depending on the current search direction) by the amount of pixels equal to the average width of a tooth on position $p_{c}$. Thus, if the last detected gap lied on position 100 and the average width of the currently searched tooth is 50 then the expected value should lie on position 50 , if the search direction is left, or 150 , if the search direction is right. $D$ is a function introduced to narrow the amount of pixels considered during every iteration of the algorithm, equal to 1 for points in the horizontal distances from the previous detected gap between $75 \%$ and $175 \%$ of the expected width of a tooth on position $p_{c}$, with the regard to the current search direction, and equal to 0 elsewhere. During every iteration the current argument maximum of the equation 3 is added to the list of gap positions and becomes the starting point of the next iteration of the algorithm. If the amount
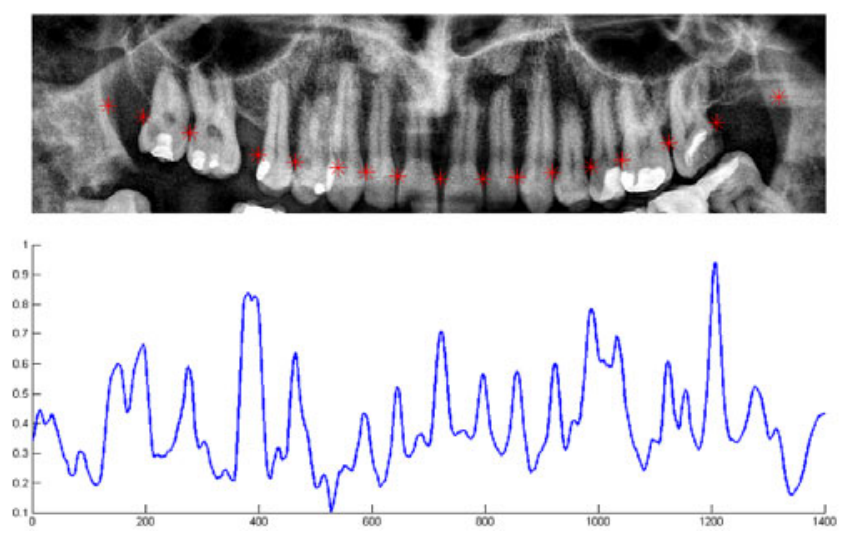

Fig. 2. Located gaps between necks of teeth for the upper jaw of the sample pantomogram (top) and the corresponding values of the pixels on the range filtered and inverted original image through which the dental pulp spline passes (bottom) 
of gaps in a given search direction for either upper or lower jaw equals 8 or the vertical edge of the image was reached, the algorithm stops. The result of this step of algorithm for the sample pantomogram is presented in Fig. 2.

Thus calculated gap locations provide a good estimation of the position of areas between teeth. However, in some cases a simple vertical line is not sufficient for the separation of two adjacent teeth. Molars and, in some cases, premolars require an additional step of the algorithm to determine the angle of the segmenting line. In order to find a straight line seperating these teeth, an additional point needs to be found between them. A greedy algorithm was used, iteratively moving 1 pixel towards the top or bottom of the radiogram, choosing the pixel in horizontal vicinity with the highest intensity on the inverted and range-filtered image and using it as the basis for the next iteration. After the number of iterations equal to half of the length of an average tooth on a pantomogram, the position of the last result becomes the second separating point. The line passing through the first and second separating point becomes the segmentation line.

\subsection{Removing the Areas Below the Roots of Teeth}

The last step is to remove the areas below the roots of teeth. It is similar to the detection of dental pulp line, i.e. the curve separating both jaws is translated vertically in search of an alignment where the sum of pixels it passes through is lower than the surrounding results, indicating that the area between the teeth line and the cheekbone line was achieved. The only difference between this part of the algorithm and the search for the line of necks of teeth is the range of translations considered during the search. After finding separating lines, every segment of the image lying between the jaws gap line, two consecutive lines separating adjacent teeth and the line below the dental roots is considered an area possibly containing a tooth and is later used in the process of feature extraction.

\section{Results and Discussion}

The described method was tested on a database containing 218 orthopantomograms and some exemplary test results are presented in this section. Firstly,

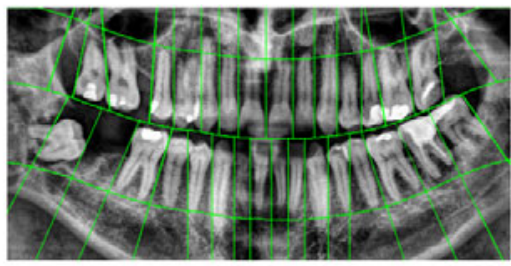

(a)

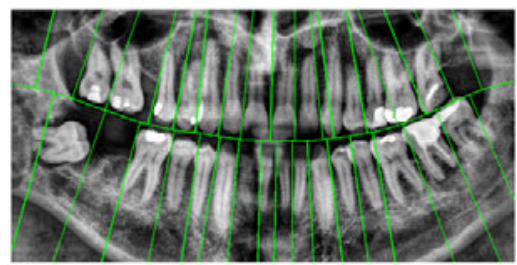

(b)

Fig. 3. A comparison of the results abtained for the method proposed in the paper (3(a) and the integral projections method described in [6] 3(b) 


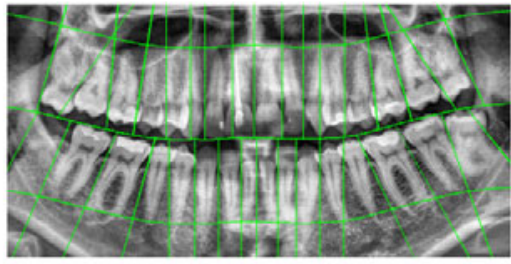

(a)

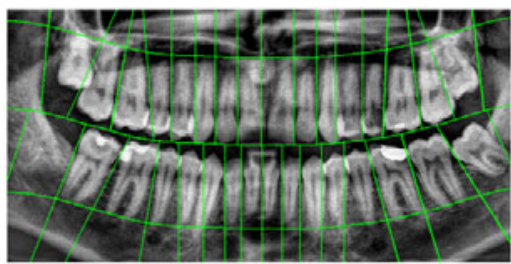

(c)

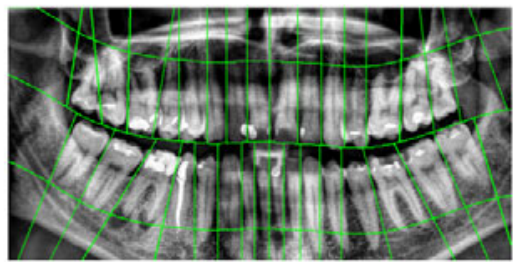

(e)

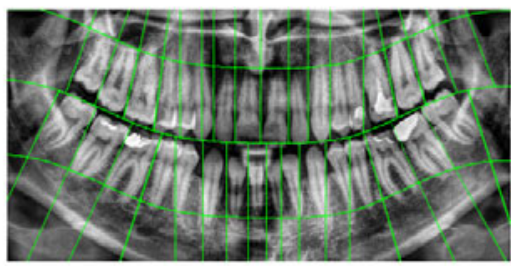

(g)

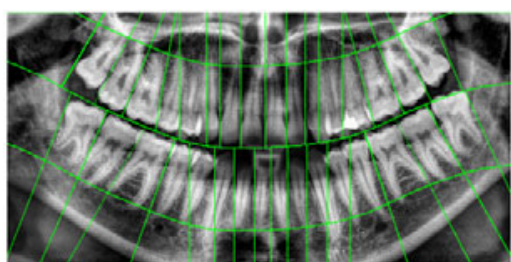

(i)

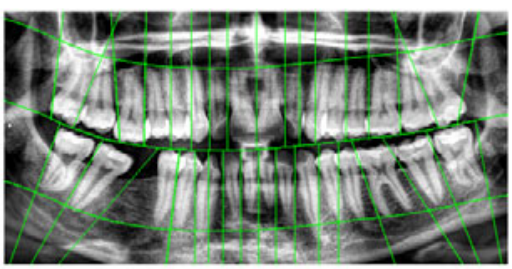

(b)

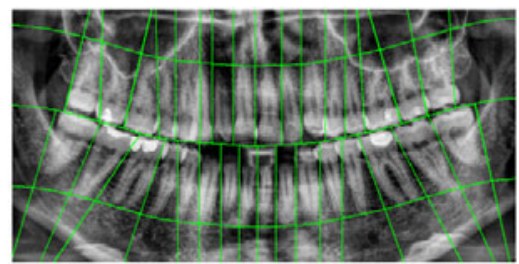

(d)

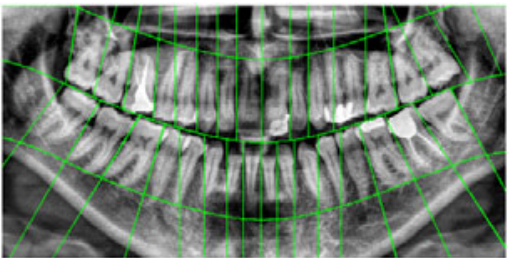

(f)

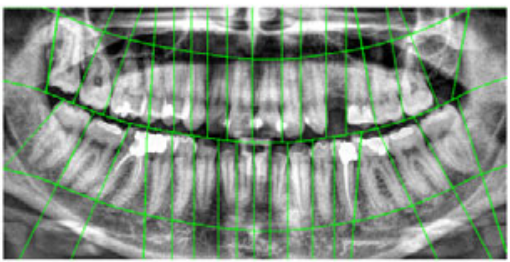

(h)

Fig. 4. Exemplary test results obtained using the presented method 
Fig. 3 presents a comparison between the results of the proposed method and the most popular approach applied so far — the integral projections ([6]). The results are comparable, with a slightly better result achieved for the proposed method - smaller number of lines passing through dental roots and the removal of the area below the roots of teeth make the segments more compact and improve the possibility of a correct shape recognition in the next step of the system. It should also be noted that during the tests the calculation of the function seen in the bottom of Fig. 2, used to detect spikes in the values indicating the gaps between teeth, took less than 1 second, whereas the calculation of the analogous function for the integral projections, i.e. the $p_{v_{i}}\left(D_{i}\right)$ in equation 1 took around 212 seconds. After these calculations, both methods use the same algorithm to detect the spikes in the resulting plot, as described in equation 3 .

Further results were presented in Fig. 4. It can be seen that the segmentation algorithm provides satisfactory results. If the teeth can be separated using a single line, the algorithm is usually able to find the optimal line of separation. The described method is also able, in some cases, to separate unerupted teeth, as can be seen in Fig. 4(c). The obvious bad results can be found in cases of occlusions so severe that it is impossible to find a straight line to separate both teeth, such as in the case of the incissor and the first premolar (respectively third and fourth tooth from the center of the image) of Fig. 4(a) and Fig. 4(e) Malaligned teeth can also be separated to a degree, but in the case of severe problems in alignment the separating line can not capture the whole tooth within a segment, for example the furthermost bottom left molar in Fig. 3(a). Detection of the ends of dental roots helps in removing bright areas of the underlying bone that would be otherwise attributed to the tooth, but in some cases a fragment of the tooth is removed as well, e.g. in Fig. 4(e). The last problem stems from the fact that dental pulp can easily be mistaken for the edge of the tooth, resulting in a mis-segmentation. This happened in the case of the lower left canine in Fig. 4(b) where the separating line passes through the middle of the tooth, but because average teeth widths are used, the final 8th tooth is separated correctly.

\section{$5 \quad$ Summary and Conclusions}

In this paper a novel method for segmenting dental panoramic radiograms into regions containing single teeth was described. It uses a different approach than the existing algorithms developed for intraoral images, focusing on detecting gaps between necks of teeth and roots of teeth, that are both easy to find on a pantomogram and allow to separate teeth even in the presence of occlusions.

The method provides satisfactory results, however some changes can be introduced in the future. A considerable improvement of its results could be achieved if a more sophisticated method was used instead of the greedy algorithm to determine the second point through which the separating line between two teeth is traced. The method could also be used as an initial step for further segmentation using for example active contours, what can improve the obtained results. 


\section{References}

1. Bowers, M.C.: Forensic Dental Evidence. Elsevier, Amsterdam (2004)

2. Lee, S., et al.: The Diversity of Dental Patterns in Orthopantomography and its Significance in Human Identification. Journal of Forensic Science 49(4), 784-786 (2004)

3. Nassar, D., Ammar, H.H.: A Prototype Automated Dental Identification System (ADIS). In: Proceedings of the 2003 Annual National Conference on Digital Government Research, pp. 1-4 (2003)

4. Abdel-Mottaleb, M., et al.: Challenges of Developing an Automated Dental Identification System. In: IEEE Mid-West Symposium for Circuits and Systems, Cairo, Egypt, pp. 411-414 (2003)

5. Fahmy, G., Nassar, D.E., Haj-Said, E., Chen, H., Nomir, O., Zhou, J., Howell, R., Ammar, H.H., Abdel-Mottaleb, M., Jain, A.K.: Towards an Automated Dental Identification System (ADIS). In: Zhang, D., Jain, A.K. (eds.) ICBA 2004. LNCS, vol. 3072, pp. 789-796. Springer, Heidelberg (2004)

6. Jain, A.K., Chen, H.: Matching of Dental X-ray Images for Human Identification. Pattern Recognition 37(7), 1519-1532 (2004)

7. Said, A., et al.: Dental X-ray Image Segmentation. In: SPIE Technologies for Homeland Security and Law Enforcement Conference (2001)

8. Zhou, J., Abdel-Mottaleb, M.: A Content-based System for Human Identification Based on Bitewing Dental X-ray Images. Pattern Recognition 38(11), 2132-2142 (2005)

9. Kass, M., Witkin, A., Terzopoulos, D.: Snakes: Active Contour Models. International Journal of Computer Vision 1(4), 321-331 (1988)

10. Frejlichowski, D., Wanat, R.: Application of the Laplacian Pyramid Decomposition to the Enhancement of Digital Dental Radiographic Images for the Automatic Person Identification. In: Campilho, A., Kamel, M. (eds.) ICIAR 2010, Part II. LNCS, vol. 6112, pp. 151-160. Springer, Heidelberg (2010) 